\title{
Dynamically controllable anisotropic metamaterials with simultaneous attenuation and amplification
}

\author{
Tom G. Mackay* \\ School of Mathematics and Maxwell Institute for Mathematical Sciences, \\ University of Edinburgh, Edinburgh EH9 3FD, Scotland, United Kingdom \\ Akhlesh Lakhtakia ${ }^{\dagger}$ \\ Nanoengineered Metamaterials Group, Department of Engineering Science and Mechanics, \\ The Pennsylvania State University, University Park, Pennsylvania 16802-6812, USA \\ (Received 18 May 2015; published 20 November 2015)
}

\begin{abstract}
Anisotropic homogeneous metamaterials that are neither wholly dissipative nor wholly active at a specific frequency are permitted by classical electromagnetic theory. Well-established homogenization formalisms indicate that such a metamaterial may be realized quite simply as a random mixture of electrically small (possibly nanoscale) spheroidal particles of at least two different isotropic dielectric materials, one of which must be dissipative but the other active. The dielectric properties of this metamaterial are influenced by the volume fraction, spatial distribution, particle shape and size, and the relative permittivities of the component materials. Similar metamaterials with more complicated linear as well as nonlinear constitutive properties are possible. Dynamic control of the active component material, for example, via stimulated Raman scattering, affords dynamic control of the metamaterial.
\end{abstract}

DOI: 10.1103/PhysRevA.92.053847

PACS number(s): 42.25.Bs, 41.20.Jb, 78.20.Ci

\section{INTRODUCTION}

Causality mandates that electromagnetic fields must attenuate as they propagate inside homogeneous passive linear materials [1]. Weak electromagnetic fields in certain spectral regimes can be amplified in some homogeneous linear materials, provided that strong electromagnetic fields can pump in the energy needed for amplification [2-5]. Conditions on the constitutive parameters of homogeneous linear materials have been derived to determine if a linear material is either dissipative or active, but not both, at a specific frequency [6].

Active component materials feature prominently in the field of metamaterials [7], in order to overcome losses [8] and to enhance performance [9]. While anisotropic structures containing active components have been reported upon previously [10-12], the prospect of simultaneous attenuation and amplification of electromagnetic fields in homogeneous materials at a specific frequency, depending upon the orientation of electric fields, has not been considered hitherto.

Materials exhibiting amplification and attenuation at the same frequency are necessarily anisotropic. A host of applications for them can be envisaged. These materials could be used in directional coupling devices [13] as well as in spatially discriminatory and/or frequency-discriminatory optical amplifiers [14]. Amplification could be dynamically controlled by exploiting, for instance, the phenomenon of stimulated Raman scattering [2,15], thereby affording dynamic control. That is, if the active component material were Raman active, then the degree of amplification achieved for a probe laser beam at a desired frequency could be dynamically

\footnotetext{
*Also at Nanoengineered Metamaterials Group, Department of Engineering Science and Mechanics, The Pennsylvania State University, University Park, PA 16802-6812, USA; T.Mackay@ed.ac.uk.

†akhlesh@psu.edu.
}

controlled by means of a strong pump laser beam at a determined frequency which induces Raman transitions. The difference between the probe and pump laser frequencies is specified by the energy levels of the Raman-active material. The ability to suppress radiation leakage in certain directions while promoting propagation in other directions could be harnessed to amplify surface-plasmon polaritons and reduce optical noise in biosensing applications [16], for example. Radomes for enhancing or reducing directionality of radiation from optical antennas could be made of these materials, leading to enhancements in the efficiency of photodetection, light emission, and sensing [17].

Motivated by these potential applications, here we propose dynamically controllable anisotropic materials which simultaneously exhibit both dissipation and amplification at a specific frequency, depending upon the orientation of electric fields.

\section{SIMULTANEOUS ATTENUATION AND AMPLIFICATION}

Consider a generally anisotropic, homogeneous, linear, dielectric material characterized by the frequency-domain constitutive relations

$$
\left.\begin{array}{l}
\mathbf{D}(\mathbf{r}, \omega)=\epsilon_{0} \underline{\underline{\epsilon}}(\omega) \cdot \mathbf{E}(\mathbf{r}, \omega) \\
\mathbf{B}(\mathbf{r}, \omega)=\mu_{0} \mathbf{H}(\mathbf{r}, \omega),
\end{array}\right\}
$$

where $\epsilon(\omega)$ is the relative permittivity dyadic $[18,19]$ at angular frequency $\omega$, and $\epsilon_{0}$ and $\mu_{0}$ are, respectively, the permittivity and permeability of free space. The time-averaged dissipated power per unit volume is given by [19]

$$
Q(\mathbf{r}, \omega)=-\frac{i \omega \epsilon_{0}}{4} \mathbf{E}^{*}(\mathbf{r}, \omega) \cdot[\underline{\underline{\epsilon}}(\omega)-\underline{\underline{\tilde{\epsilon}}}(\omega)] \cdot \mathbf{E}(\mathbf{r}, \omega),
$$


where $i=\sqrt{-1}$; an $\exp (-i \omega t)$ dependence on time $t$ is implicit; the superscript * denotes the complex conjugate; and $\underline{\underline{\tilde{\epsilon}}}(\omega)$ is the Hermitian conjugate of $\underline{\underline{\epsilon}}(\omega)$.

At a specific angular frequency $\omega$, the chosen material is classified as [20] (1) dissipative if $Q(\mathbf{r}, \omega)>0$, which requires the dyadic $i[\underline{\epsilon}(\omega)-\tilde{\epsilon}(\omega)]$ to be negative definite, or (2) active if $Q(\mathbf{r}, \omega)<\overline{0}$, which requires the dyadic $i[\underline{\epsilon}(\omega)-\underline{\tilde{\epsilon}}(\omega)]$ to be positive definite. All eigenvalues of a negative or positive definite dyadic are negative or positive [21]. Henceforth, for compact representation, the dependencies of $Q$ and $\mathbf{E}$ on $\omega$ and $\mathbf{r}$ will not be explicitly stated; similarly, the dependency of $\epsilon$ (and its components) on $\omega$ will not be explicitly stated.

$\overline{\bar{T}}$ he foregoing classification fails to accommodate to the prospect of $i(\underline{\epsilon}-\tilde{\tilde{\epsilon}})$ being indefinite [21], i.e., when some but not all eigenvalues of $i(\underline{\epsilon}-\underline{\tilde{\epsilon}})$ are positive, the remaining eigenvalues being negative. For orthorhombic materials [22], $i(\underline{\epsilon}-\underline{\tilde{\epsilon}})$ is indefinite provided that $\operatorname{Im}\{\underline{\epsilon}\}$ is indefinite, where the operator $\operatorname{Im}\{\cdot\}$ delivers the imaginary part.

The simplest material, at least from a mathematical perspective, for which $\operatorname{Im}\{\underline{\epsilon}\}$ is indefinite is a uniaxial dielectric material the relative permittivity dyadic of which has the form [19]

$$
\underline{\underline{\epsilon}}=\epsilon^{\perp}(\underline{\underline{I}}-\hat{\mathbf{u}} \hat{\mathbf{u}})+\epsilon^{\|} \hat{\mathbf{u}} \hat{\mathbf{u}},
$$

with $\operatorname{Im}\left\{\epsilon^{\perp}\right\} \operatorname{Im}\left\{\epsilon^{\|}\right\}<0$. Herein $\underline{I}$ is the identity dyadic and the unit vector $\hat{\mathbf{u}}$ is parallel to the material's optic axis. For this material, it may be inferred from Eq. (2) that $Q$ for $\mathbf{E}$ directed along $\hat{\mathbf{u}}$ has the opposite sign to $Q$ for $\mathbf{E}$ directed perpendicular to $\hat{\mathbf{u}}$, because

$$
Q=\frac{\omega \epsilon_{0}}{2}\left[\operatorname{Im}\left\{\epsilon^{\perp}\right\} \mathbf{E}^{*} \cdot(\underline{\mathbf{I}}-\hat{\mathbf{u}} \hat{\mathbf{u}}) \cdot \mathbf{E}+\operatorname{Im}\left\{\epsilon^{\|}\right\} \mathbf{E}^{*} \cdot \hat{\mathbf{u}} \hat{\mathbf{u}} \cdot \mathbf{E}\right] .
$$

Thus, there is dissipation associated with certain orientations of the electric field but amplification with other orientations.

\section{REALIZATION AS A HOMOGENIZED COMPOSITE MATERIAL}

Is it possible to realize a uniaxial dielectric material for which $\operatorname{Im}\{\underline{\epsilon}\}$ is indefinite? We now demonstrate, using well-established theoretical formalisms based on the homogenization of particulate composite materials, that materials with indefinite $\operatorname{Im}\{\underline{\epsilon}\}$ may be conceptualized as homogenized composite materials (HCMs). As these engineered materials will simultaneously exhibit both amplification and dissipation at the same frequency, they should more properly be called metamaterials [23]. In the visible spectrum $(380-770 \mathrm{THz})$, the maximum linear dimensions of the component particles are required to be less than $\sim 30 \mathrm{~nm}$ [24].

Consider a composite material that is a mixture of two component materials labeled "a" and "b." Dispersed randomly as identically oriented, electrically small, conformal spheroids, both component materials are isotropic dielectric materials with relative permittivities $\epsilon_{a}$ and $\epsilon_{b}$. The surface of a spheroid, relative to its centroid, is prescribed by the position vector $\mathbf{r}=\rho \underline{\underline{U}} \cdot \hat{\mathbf{r}}_{s}$, where $\rho>0$ is a linear measure of particle size, the shape dyadic

$$
\underline{\underline{U}}=\frac{1}{\sqrt{U}}(\underline{\underline{I}}-\hat{\mathbf{u}} \hat{\mathbf{u}})+U \hat{\mathbf{u}} \hat{\mathbf{u}} \quad(U>0)
$$

contains the shape parameter $U$, and the unit vector $\hat{\mathbf{r}}_{s}$ prescribes the surface of the concentric unit sphere. Oblate spheroidal particles are characterized by $U \in(0,1)$, prolate spheroidal particles are characterized by $U>1$, and spherical particles are characterized by $U=1$. The volume fraction of component material " $a$ " is $f_{a}$, while that of component material " $\mathrm{b}$ " is $f_{b}=1-f_{a}$. The spheroidal shape and the identical alignment of the component particles endows the HCM with uniaxial symmetry $[18,19]$; i.e., the relative permittivity dyadic of the HCM has the form

$$
\underline{\epsilon}_{\mathrm{HCM}}=\epsilon_{\mathrm{HCM}}^{\perp}(\underline{\underline{I}}-\hat{\mathbf{u}} \hat{\mathbf{u}})+\epsilon_{\mathrm{HCM}}^{\|} \hat{\mathbf{u}} \hat{\mathbf{u}} .
$$

Porous columnar-thin-film sections and nanoparticle arrays can be fabricated to realize such HCMs $[25,26]$.

\section{ESTIMATES OF CONSTITUTIVE PARAMETERS}

Let us now present representative numerical estimates of $\stackrel{\epsilon}{\mathrm{HCM}}_{\text {}}$, as yielded by the Bruggeman formalism [27], the strong-permittivity-fluctuation theory (SPFT) [28,29], and the Maxwell Garnett formalism [30,31]; these estimates are identified by replacing the subscripts "HCM" by "Br," "SPFT," and "MG," respectively.

For the purpose of illustration, suppose that the component material "a" is an active material with $\epsilon_{a}=2-0.03 i$. This value of $\epsilon_{a}$ lies comfortably within the range typically employed for active components of metamaterials in the visible regime. For example, a mixture of two commonly used amplification materials, namely, Rhodamine 800 and Rhodamine $6 \mathrm{G}$, possesses a relative permittivity with imaginary part in the range $(-0.15,-0.02)$ and real part in the range $(1.8,2.3)$ across the frequency range $440-500 \mathrm{THz}$, depending upon the relative concentrations and the external pumping rate [11]. Component material " $b$ " is taken to be a dissipative material specified by $\epsilon_{b}=3+0.05 i$, and the shape parameter $U=5$. The real and imaginary parts of the HCM's relative-permittivity scalars $\epsilon_{\mathrm{Br}}^{\perp}$ and $\epsilon_{\mathrm{Br}}^{\|}$, as estimated using the Bruggeman formalism [27], are plotted against the volume fraction $f_{a}$ in Fig. 1 . The real parts of $\epsilon_{\mathrm{Br}}^{\perp}$ and $\epsilon_{\mathrm{Br}}^{\|}$decrease in an approximately linear manner from three to two as $f_{a}$ increases from zero to one. Both $\operatorname{Im}\left\{\epsilon_{\mathrm{Br}}^{\perp}\right\}$ and $\operatorname{Im}\left\{\epsilon_{\mathrm{Br}}^{\|}\right\}$decrease uniformly from $\operatorname{Im}\left\{\epsilon_{b}\right\}$ to $\operatorname{Im}\left\{\epsilon_{a}\right\}$ as $f_{a}$ increases from zero to one, but the decrease in $\operatorname{Im}\left\{\epsilon_{\mathrm{Br}}^{\perp}\right\}$ is more distinctly nonlinear than that in $\operatorname{Im}\left\{\epsilon_{\mathrm{Br}}^{\|}\right\}$. The data show the following.

(i) For $0<f_{a}<0.52$, the HCM exhibits only dissipation, regardless of the orientation of the electric field $\mathbf{E}$.

(ii) For $0.63<f_{a}<1$, the HCM exhibits only amplification, regardless of the orientation of $\mathbf{E}$.

(iii) For $0.52<f_{a}<0.63$, the HCM exhibits simultaneously both dissipation and amplification, depending on the orientation of $\mathbf{E}$.

Parenthetically, the HCM represented in Fig. 1 is not an "indefinite" material [18] since $\operatorname{Re}\left\{\underline{\epsilon}_{\mathrm{HCM}}\right\}$ is positive definite.

The shape of the component particles plays an important role in the simultaneous exhibition of dissipation and 

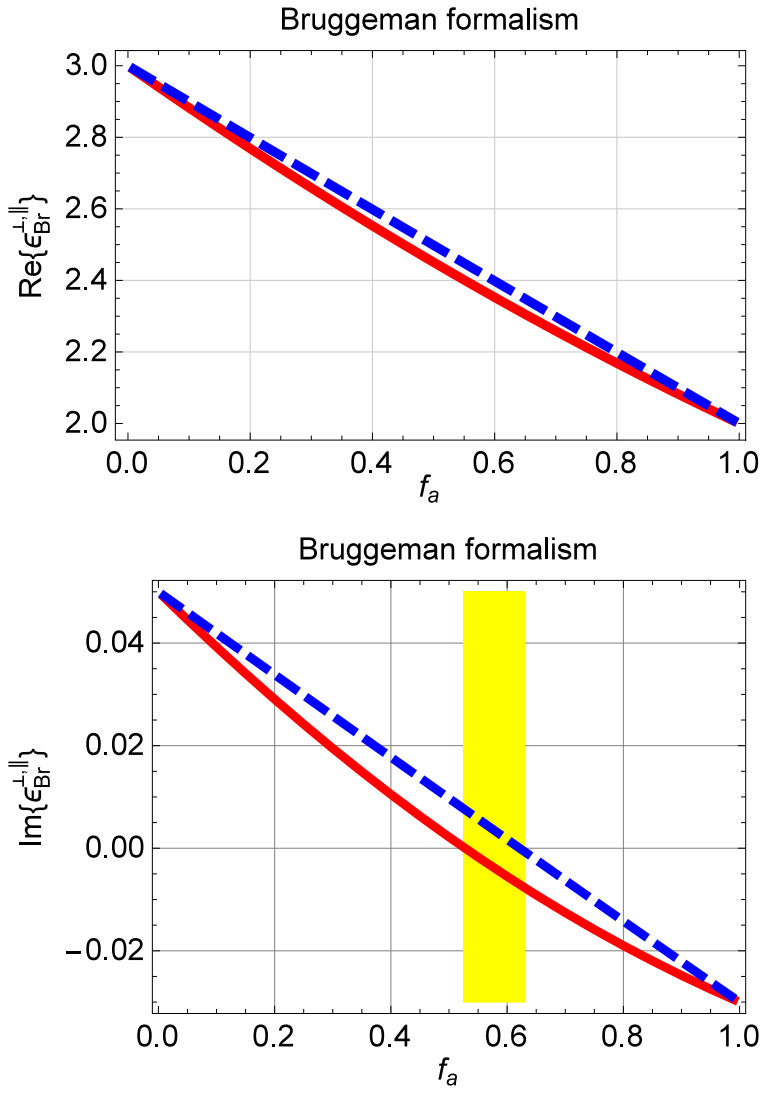

FIG. 1. (Color online) Real and imaginary parts of $\epsilon_{\mathrm{Br}}^{\perp}$ (red, solid curves) and $\epsilon_{\mathrm{Br}}^{\|}$(blue, dashed curves) plotted against the volume fraction $f_{a}$, when $\epsilon_{a}=2-0.03 i, \epsilon_{b}=3+0.05 i$, and $U=5$. The yellow rectangle indicates the $f_{a}$ range where $\operatorname{Im}\left\{\epsilon_{\mathrm{Br}}^{\perp}\right\}<0$ but $\operatorname{Im}\left\{\epsilon_{\mathrm{Br}}^{\|}\right\}>0$.

amplification. In Fig. 2, the real and imaginary parts of $\epsilon_{\mathrm{Br}}^{\perp}$ and $\epsilon_{\mathrm{Br}}^{\|}$are plotted against $U$, when $f_{a}=0.535$ and the relative permittivities of the component materials are the same as for Fig. 1. The component particles are oblate spheroids for $U<1$, prolate spheroids for $U>1$, and spheres for $U=1$. Both the real and imaginary parts of $\epsilon_{\mathrm{Br}}^{\perp}$ decrease nonlinearly as $U$ increases whereas both the real and imaginary parts of $\epsilon_{\mathrm{Br}}^{\|}$increase nonlinearly as $U$ increases, with $\epsilon_{\mathrm{Br}}^{\perp}=\epsilon_{\mathrm{Br}}^{\|}$at $U=1$. In the vicinity of $U=1, \operatorname{Im}\left\{\epsilon_{\mathrm{Br}}^{\perp}\right\}$ and $\operatorname{Im}\left\{\epsilon_{\mathrm{Br}}^{\|}\right\}$are both positive. However, for $U<0.75$, we find that $\operatorname{Im}\left\{\epsilon_{\mathrm{Br}}^{\|}\right\}<0$ but $\operatorname{Im}\left\{\epsilon_{\mathrm{Br}}^{\perp}\right\}>0$. Incidentally, the Bruggeman formalism for a particulate composite material yields the same results in the limit $U \rightarrow 0$ as for a periodically laminated composite material [32]. Also, for $U>2.4$, we find that $\operatorname{Im}\left\{\epsilon_{\mathrm{Br}}^{\|}\right\}>0$ but $\operatorname{Im}\left\{\epsilon_{\mathrm{Br}}^{\perp}\right\}<0$. Thus, the HCM exhibits simultaneously both dissipation and amplification provided that the component spheroidal particles are either sufficiently flattened or sufficiently elongated.

The effect of the relative permittivity of the active component material on the simultaneous exhibition of dissipation and amplification is taken up through Fig. 3. Therein, the real and imaginary parts $\epsilon_{\mathrm{Br}}^{\perp}$ and $\epsilon_{\mathrm{Br}}^{\|}$, as well as the product $\operatorname{Im}\left\{\epsilon_{\mathrm{Br}}^{\perp}\right\} \operatorname{Im}\left\{\epsilon_{\mathrm{Br}}^{\|}\right\}$, are plotted against the real and (negative) imaginary parts of $\epsilon_{a}$, the product being negative if and only if
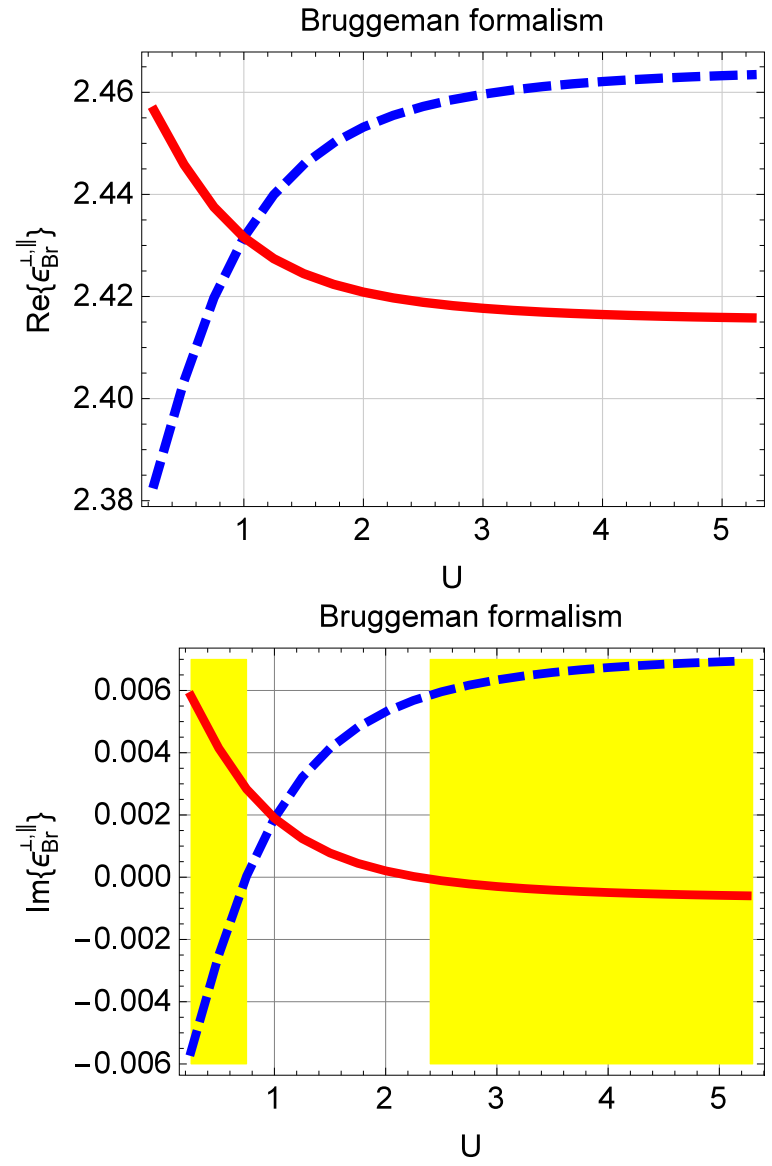

FIG. 2. (Color online) Real and imaginary parts of $\epsilon_{\mathrm{Br}}^{\perp}$ (red, solid curves) and $\epsilon_{\mathrm{Br}}^{\|}$(blue, dashed curves) plotted against the shape parameter $U$, when $\epsilon_{a}=2-0.03 i, \epsilon_{b}=3+0.05 i$, and $f_{a}=0.535$. The two yellow rectangles indicate the $U$ ranges where the product $\operatorname{Im}\left\{\epsilon_{\mathrm{Br}}^{\perp}\right\} \operatorname{Im}\left\{\epsilon_{\mathrm{Br}}^{\|}\right\}<0$.

$\operatorname{Im}\left\{\epsilon_{\mathrm{Br}}^{\perp}\right\}$ and $\operatorname{Im}\left\{\epsilon_{\mathrm{Br}}^{\|}\right\}$have opposite signs. For calculating these results, we fixed $\epsilon_{b}=3+0.05 i, f_{a}=0.25$, and $U=5$. The $\epsilon_{a}$ regime for which the HCM exhibits simultaneously both amplification and attenuation is characterized by relatively small values of $\operatorname{Re}\left\{\epsilon_{a}\right\}$ and relatively large values of $-\operatorname{Im}\left\{\epsilon_{a}\right\}$. Indeed, as $-\operatorname{Im}\left\{\epsilon_{a}\right\}$ approaches zero, the HCM is either exclusively dissipative or exclusively active, this state being attained at larger values of $-\operatorname{Im}\left\{\epsilon_{a}\right\}$ as $\operatorname{Re}\left\{\epsilon_{a}\right\}$ approaches two.

Next let us turn to a more sophisticated homogenization formalism: the SPFT [33]. Unlike the Bruggeman formalism, the SPFT can accommodate a comprehensive description of the distributional statistics of the component materials, via the characteristic functions

$$
\Phi_{\ell}(\mathbf{r})=\left\{\begin{array}{ll}
1, & \mathbf{r} \in V_{\ell} \\
0, & \mathbf{r} \notin V_{\ell}
\end{array} \quad \ell \in\{a, b\} .\right.
$$

Herein, the regions occupied by the component materials " $a$ " and "b" are identified by $V_{a}$ and $V_{b}$, respectively. The ensemble average $\left\langle\Phi_{\ell}(\mathbf{r})\right\rangle_{e}$ equals the volume fraction $f_{\ell}, \ell \in\{a, b\}$. In the usual implementation of the bilocally approximated SPFT [33], the distributional statistics of the component 

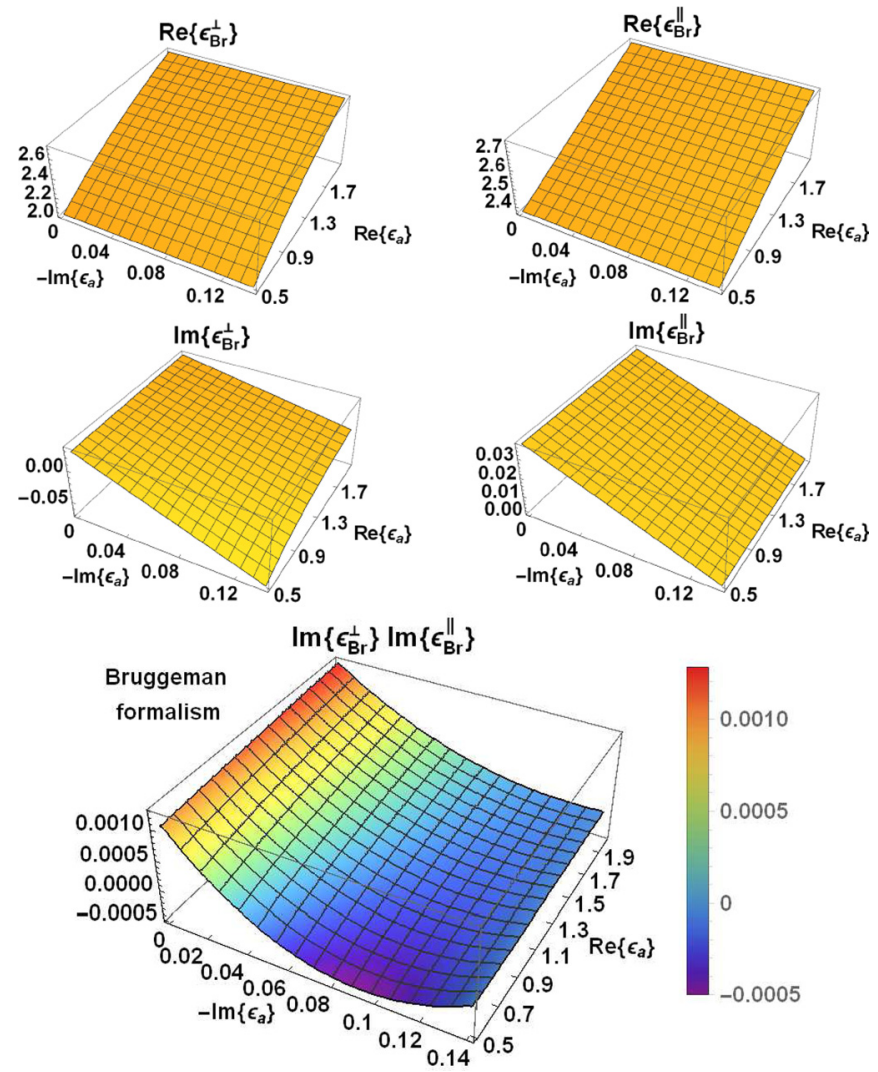

FIG. 3. (Color online) Real and imaginary parts $\epsilon_{\mathrm{Br}}^{\perp}$ and $\epsilon_{\mathrm{Br}}^{\|}$, as well as the product $\operatorname{Im}\left\{\epsilon_{\mathrm{Br}}^{\perp}\right\} \operatorname{Im}\left\{\epsilon_{\mathrm{Br}}^{\|}\right\}$, plotted against the real and (negative) imaginary parts of the relative permittivity $\epsilon_{a}$, when $\epsilon_{b}=3+0.05 i, f_{a}=0.25$, and $U=5$.

materials are characterized by the second moment

$$
\left\langle\Phi_{\ell}(\mathbf{r}) \Phi_{\ell}\left(\mathbf{r}^{\prime}\right)\right\rangle_{e}= \begin{cases}f_{\ell}, & \left|\underline{\underline{U}^{-1}} \cdot\left(\mathbf{r}-\mathbf{r}^{\prime}\right)\right| \leqslant L \\ f_{\ell}^{2}, & \underline{\underline{U^{-1}}} \cdot\left(\mathbf{r}-\mathbf{r}^{\prime}\right) \mid>L\end{cases}
$$

for $\ell \in\{a, b\}$, with $L$ as the correlation length.

The real and imaginary parts of the HCM's relativepermittivity scalars $\epsilon_{\mathrm{SPFT}}^{\perp}$ and $\epsilon_{\mathrm{SPFT}}^{\|}$, as estimated using the bilocally approximated SPFT, are plotted against the normalized correlation length $k_{0} L$ in Fig. 4 , where $k_{0}=$ $\omega \sqrt{\epsilon_{0} \mu_{0}}$. For these calculations, we set $\epsilon_{a}=2-0.03 i, \epsilon_{b}=$ $3+0.05 i, f_{a}=0.535$, and $U=5$; also, we used an extended version of the SPFT [34] which explicitly accommodates the particle-size parameter $\rho$. Results are presented in Fig. 4 for $\rho \in\{0,0.5 L, L\}$. In this figure, the real and imaginary parts of both $\epsilon_{\mathrm{SPFT}}^{\perp}$ and $\epsilon_{\mathrm{SPFT}}^{\|}$increase uniformly as $k_{0} L$ increases, for all values of $\rho / L$ considered. Also, $\operatorname{Im}\left\{\epsilon_{\mathrm{SPFT}}^{\|}\right\}>0$ for all values of $k_{0} L$ and $\rho / L$. However, $\operatorname{Im}\left\{\epsilon_{\mathrm{SPFT}}^{\perp}\right\}<0$ for low values of $k_{0} L$ while $\operatorname{Im}\left\{\epsilon_{\mathrm{SPFT}}^{\perp}\right\}>0$ for high values of $k_{0} L$. Furthermore, the transition from negative $\operatorname{Im}\left\{\epsilon_{\mathrm{SPFT}}^{\perp}\right\}$ to positive $\operatorname{Im}\left\{\epsilon_{\mathrm{SPFT}}^{\perp}\right\}$ occurs at lower values of $k_{0} L$ when the size parameter $\rho$ is larger. Thus, the HCM exhibits simultaneously both amplification and dissipation provided that both $L$ and $\rho$ are sufficiently small.

Last, we present estimates of the HCM's relative permittivity dyadic provided by the Maxwell Garnett formalism [27], whose provenance is quite different from that of either the
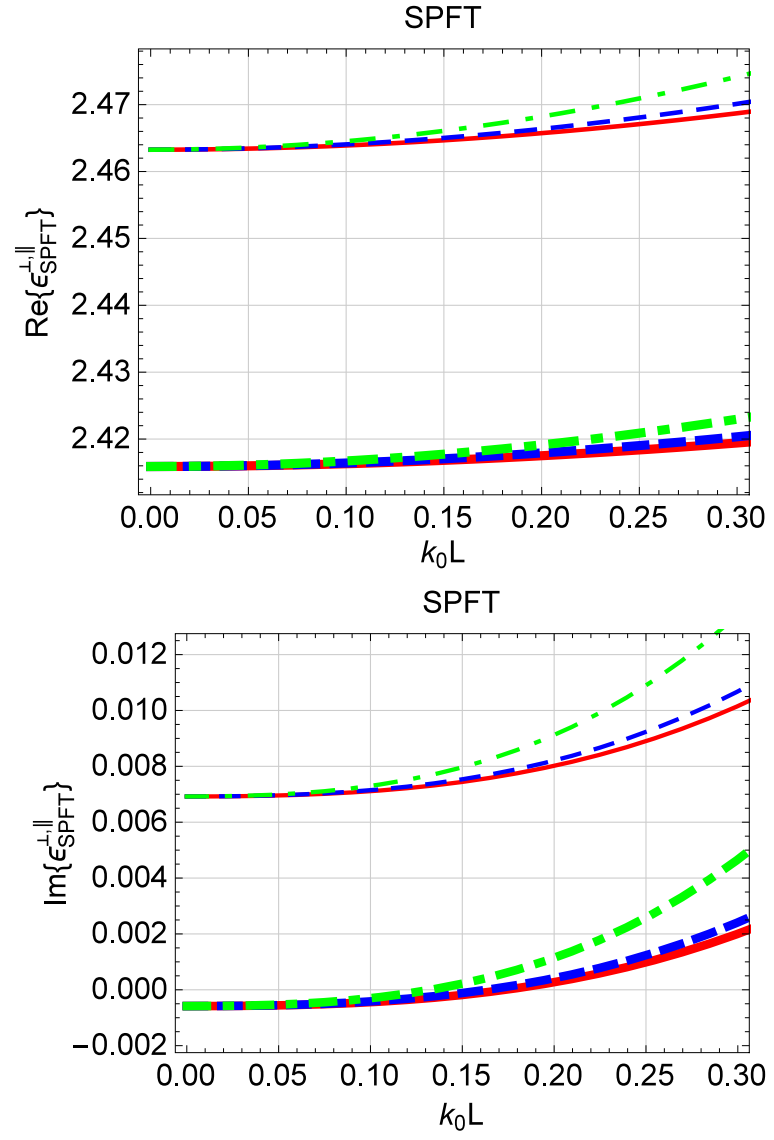

FIG. 4. (Color online) Real and imaginary parts of $\epsilon_{\mathrm{SPFT}}^{\perp}$ (thick curves) and $\epsilon_{\mathrm{SPFT}}^{\|}$(thin curves) plotted against the normalized correlation length $k_{0} L$, when $\epsilon_{a}=2-0.03 i, \epsilon_{b}=3+0.05 i, f_{a}=$ 0.535 , and $U=5$. The size parameter $\rho=0$ (red, solid curves), $0.5 L$ (blue, dashed curves), and $L$ (green, broken dashed curves).

Bruggeman formalism or the SPFT. We consider that particles of component material "a" are dispersed randomly in the component material "b." The Maxwell Garnett formalism is restricted to dilute composite materials $\left(f_{a} \lesssim 0.3\right)$. The real and imaginary parts $\epsilon_{\mathrm{MG}}^{\perp}$ and $\epsilon_{\mathrm{MG}}^{\|}$, as well as the product $\operatorname{Im}\left\{\epsilon_{\mathrm{MG}}^{\perp}\right\} \operatorname{Im}\left\{\epsilon_{\mathrm{MG}}^{\|}\right\}$, are plotted against the real and (negative) imaginary parts of $\epsilon_{a}$ in Fig. 5. The component materials are as specified for Fig. 3, with one exception: the shape parameter $U$ applies only to component material "a" since particle shape is irrelevant to the host material in the Maxwell Garnett formalism. The extent of the $\epsilon_{a}$ regime for which $\operatorname{Im}\left\{\epsilon_{\mathrm{MG}}^{\perp}\right\} \operatorname{Im}\left\{\epsilon_{\mathrm{MG}}^{\|}\right\}<0$ in Fig. 5 is slightly smaller than of the analogous $\epsilon_{a}$ regime in Fig. 3. But, other than this relatively minor difference, the plots of $\operatorname{Im}\left\{\epsilon_{\mathrm{MG}}^{\perp}\right\} \operatorname{Im}\left\{\epsilon_{\mathrm{MG}}^{\|}\right\}$in Fig. 5 and of $\operatorname{Im}\left\{\epsilon_{\mathrm{Br}}^{\perp}\right\} \operatorname{Im}\left\{\epsilon_{\mathrm{Br}}^{\|}\right\}$in Fig. 3 are very similar.

The estimates of $\underline{\epsilon}_{\mathrm{HCM}}$ provided by the Bruggeman and Maxwell Garnett formalisms-as represented in Figs. 1, 2, 3, and 5-are in close agreement over the volume-fraction range appropriate to the Maxwell Garnett formalism (i.e., $0<f_{a} \lesssim 0.3$ ). The estimates provided by the two formalisms are identical in the limit $f_{a} \rightarrow 0$ and very small differences emerge as $f_{a}$ increases. Indeed, the corresponding plots of the real and imaginary parts of the components of $\underline{\underline{\epsilon r}}_{\mathrm{Br}}$ and $\underline{\underline{\epsilon}}_{\mathrm{MG}}$ 

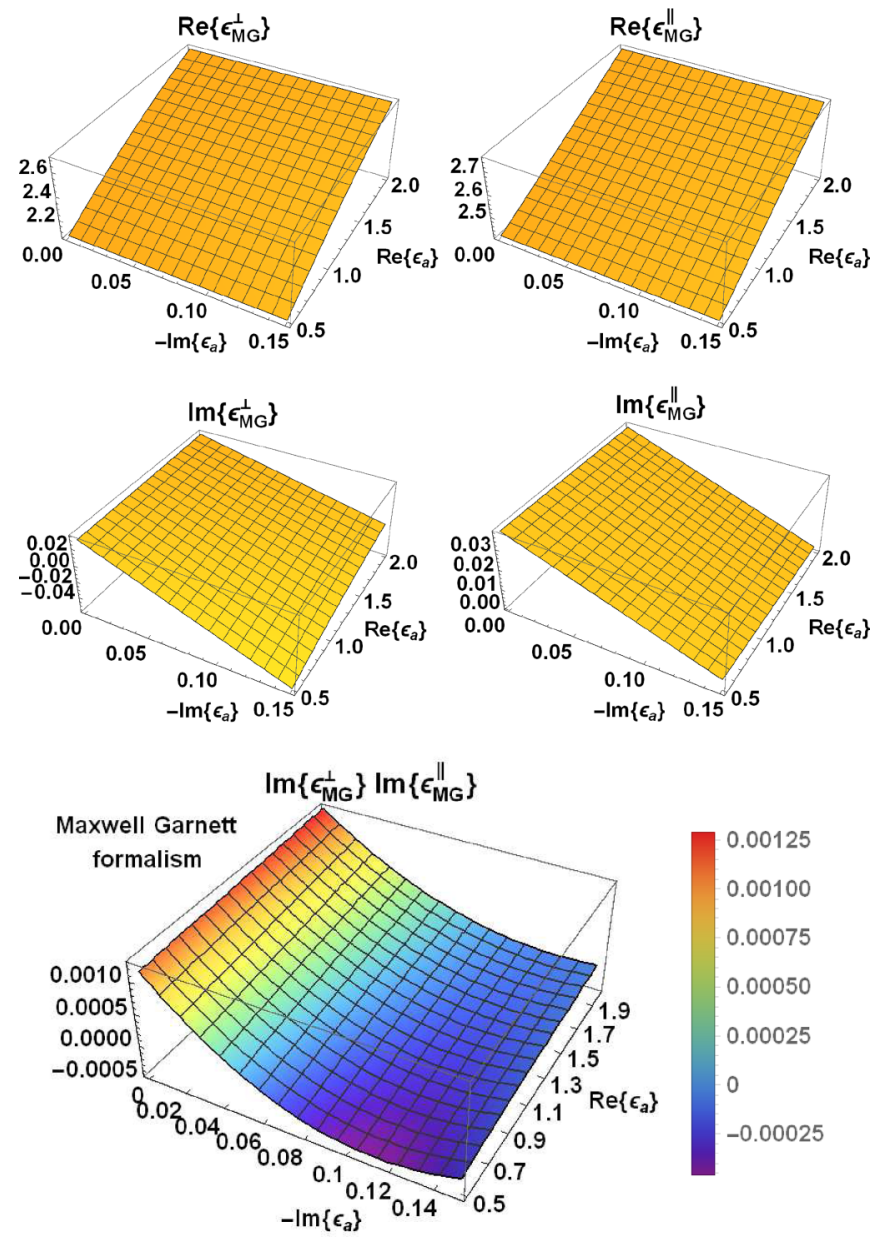

FIG. 5. (Color online) Real and imaginary parts $\epsilon_{\mathrm{MG}}^{\perp}$ and $\epsilon_{\mathrm{MG}}^{\|}$, as well as the product $\operatorname{Im}\left\{\epsilon_{\mathrm{MG}}^{\perp}\right\} \operatorname{Im}\left\{\epsilon_{\mathrm{MG}}^{\|}\right\}$, plotted against the real and (negative) imaginary parts of the relative permittivity $\epsilon_{a}$, when $\epsilon_{b}=$ $3+0.05 i, f_{a}=0.25$, and $U=5$. Particles of component material "a" are taken to be dispersed randomly in the component material "b," and the shape parameter $U$ applies only to component material "a."

in Figs. 3 and 5, respectively, are almost indistinguishable to the naked eye. These estimates are also in close agreement with the corresponding estimates provided by the bilocally approximated SPFT, as represented in Fig. 4. The imaginary parts of the components of $\underline{\epsilon}_{\mathrm{HCM}}$ estimated by the SPFT deviate slightly from their corresponding Bruggeman and Maxwell Garnett counterparts, with the deviation increasing in magnitude as the correlation length $L$ and the size parameter $\rho$ increase. This deviation reflects the fact that the SPFT formalism accommodates coherent scattering losses via $L$ and $\rho$ [33], whereas the Bruggeman and Maxwell Garnett formalisms do not.

Rigorous theoretical bases have been firmly established for each of the three homogenization formalisms employed here [27-31,33]. Techniques based on such homogenization formalisms have the advantages over full-wave numerical techniques, based on the finite-element method or the finitedifference time-domain method [35-37], for example, that they provide estimates of the constitutive parameters which are independent of the shape and size of the bulk material involved, and these estimates apply for all possible incident fields with sources not located in the bulk material. However, it is important to bear in mind that the predictions of constitutive parameters provided by any homogenization formalism are estimates. The ultimate checks on such estimates can only be provided by careful experimental studies.

\section{SIMULTANEOUS ATTENUATION AND AMPLIFICATION EXEMPLIFIED}

Power flow associated with electromagnetic propagation is represented by the time-averaged Poynting vector $\mathbf{P}=$ $(1 / 2) \operatorname{Re}\left\{\mathbf{E} \times \mathbf{H}^{*}\right\}$, with the operator $\operatorname{Re}\{\cdot\}$ delivering the real part. Since $\nabla \cdot \mathbf{P}=-Q$ in a region devoid of externally impressed sources [1,6], amplification and dissipation should be discernible through plane-wave propagation.

Suppose that the direction of propagation is parallel to the unit vector â. An ordinary plane wave will propagate in the chosen $\mathrm{HCM}$ with wave number $k_{\mathrm{or}}=k_{0} \sqrt{\epsilon_{\mathrm{HCM}}^{\perp}}$, regardless of the angle $\theta=\cos ^{-1}(\hat{\mathbf{a}} \cdot \hat{\mathbf{u}})$. An extraordinary plane wave will propagate in the chosen HCM with wave number

$$
k_{\mathrm{ex}}=k_{0} \sqrt{\frac{\epsilon_{\mathrm{HCM}}^{\perp} \epsilon_{\mathrm{HCM}}^{\|}}{\epsilon_{\mathrm{HCM}}^{\perp} \sin ^{2} \theta+\epsilon_{\mathrm{HCM}}^{\|} \cos ^{2} \theta}}
$$

that does depend on $\theta$.

Consider two examples.

(i) When $f_{a}=0.6$ in Fig. $1, \epsilon_{\mathrm{Br}}^{\perp}=2.353-0.006 i$ and $\epsilon_{\mathrm{Br}}^{\|}=2.398+0.002 i$, which yield $k_{\mathrm{or}}=(1.534-0.002 i) k_{0}$ with $\operatorname{Re}\left\{k_{\text {ex }}\right\}>0$ for all $\theta$ and $\operatorname{Im}\left\{k_{\text {ex }}\right\}>0$ for $60.5^{\circ}<\theta<$ $119.5^{\circ}$. Hence, the ordinary plane wave is amplified for all propagation directions while the extraordinary plane wave is amplified for $\theta \in\left(0^{\circ}, 60.5^{\circ}\right) \cup\left(119.5^{\circ}, 180^{\circ}\right)$ but attenuated for $\theta \in\left(60.5^{\circ}, 119.5^{\circ}\right)$.

(ii) When $U=0.25$ in Fig. $2, \epsilon_{\mathrm{Br}}^{\perp}=2.456+0.006 i$ and $\epsilon_{\mathrm{Br}}^{\|}=2.383-0.006 i$, which yield $k_{\mathrm{or}}=(1.567+0.002 i) k_{0}$ with $\operatorname{Re}\left\{k_{\text {ex }}\right\}>0$ for all $\theta$ and $\operatorname{Im}\left\{k_{\text {ex }}\right\}<0$ for $44.1^{\circ}<$ $\theta<135.9^{\circ}$. Hence, the ordinary plane wave is attenuated for all propagation directions while the extraordinary plane wave is amplified for $\theta \in\left(44.1^{\circ}, 135.9^{\circ}\right)$ but attenuated for $\theta \in\left(0^{\circ}, 44.1^{\circ}\right) \cup\left(135.9^{\circ}, 180^{\circ}\right)$.

\section{CLOSING REMARKS}

In conclusion, according to the estimates afforded by three different well-established homogenization formalisms, an HCM that exhibits simultaneously attenuation and amplification of electromagnetic fields at a specific frequency may be realized quite simply as a random mixture of electrically small spheroids of two different materials. Both component materials are isotropic dielectric materials, one of which is dissipative while the other is active. The realization of such an HCM depends upon the volume fraction, spatial distribution, particle shape and size, and the relative permittivities of the component materials. Dynamic control of the active component material, for example, via stimulated Raman scattering, affords dynamical control of the HCM. Thus, a class of metamaterials is proposed which are neither wholly dissipative nor wholly active. Although we have illustrated the concept with uniaxial 
dielectric HCMs, particulate composite materials with more complicated linear and/or nonlinear constitutive properties and displaying both dissipation and amplification at the same frequency can be designed [18]. Finally, even periodically laminated composite materials [32] offer similar promise, but particulate composite materials may be more readily fabricated than their laminar counterparts.

\section{ACKNOWLEDGMENTS}

T.G.M. acknowledges the support of Engineering and Physical Sciences Research Council Grant No. EP/M018075/1. A.L. thanks the Charles Godfrey Binder Endowment at The Pennsylvania State University for partial financial support of his research activities.
[1] A. Zangwill, Modern Electrodynamics (Cambridge University Press, New York, 2013).

[2] A. Yariv, in Quantum Electronics, 3rd ed. (Wiley, New York, 1989), p. 466.

[3] V. G. Bespalov and N. S. Makarov, Opt. Spectrosc. 95, 442 (2003).

[4] D. Dimitropoulos, V. Raghunathan, R. Claps, and B. Jalali, Opt. Express 12, 149 (2004).

[5] D. R. Solli, P. Koonath, and B. Jalali, Phys. Rev. A 79, 053853 (2009).

[6] J. A. Kong, in Electromagnetic Wave Theory (Wiley, New York, 1985), pp. 50-51.

[7] O. Hess, J. B. Pendry, S. A. Maier, R. F. Oulton, J. M. Hamm, and K. L. Tsakmakidis, Nat. Mater. 11, 573 (2012).

[8] Z.-G. Dong, H. Liu, T. Li, Z.-H. Zhu, S.-M. Wang, J.-X. Cao, S.-N. Zhu, and X. Zhang, Appl. Phys. Lett. 96, 044104 (2010).

[9] G. Strangi, A. De Luca, S. Ravaine, M. Ferrie, and R. Bartolino, Appl. Phys. Lett. 98, 251912 (2011).

[10] S. A. Tretyakov and T. G. Kharina, Electromagnetics 20, 155 (2000).

[11] L. Sun, X. Yang, and J. Gao, Appl. Phys. Lett. 103, 201109 (2013).

[12] R. S. Savelev, I. V. Shadrivov, P. A. Belov, N. N. Rosanov, S. V. Fedorov, A. A. Sukhorukov, and Y. S. Kivshar, Phys. Rev. B 87, 115139 (2013).

[13] R. K. Mongia, I. J. Bahl, P. Bhartia, and J. Hong, RF and Microwave Coupled-Line Circuits, 2nd ed. (Artech House, Norwood, MA, 2007).

[14] M. Premaratne and G. P. Agrawal, Light Propagation in Gain Media: Optical Amplifiers (Cambridge University Press, Cambridge, England, 2011).

[15] A. Lakhtakia and S. A. Ramakrishna, J. Opt. (UK) 12, 085101 (2010).

[16] I. De Leon and P. Berini, Nat. Photon. 4, 382 (2010).

[17] L. Novotny and N. van Hulst, Nat. Photon. 5, 83 (2011).
[18] T. G. Mackay and A. Lakhtakia, Electromagnetic Anisotropy and Bianisotropy: A Field Guide (Word Scientific, Singapore, 2010).

[19] H. C. Chen, Theory of Electromagnetic Waves (McGraw-Hill, New York, 1983).

[20] E. L. Tan, Microw. Opt. Technol. Lett. 41, 133 (2004).

[21] H. Lütkepohl, Handbook of Matrices (Wiley, New York, 1996).

[22] J. F. Nye, Physical Properties of Crystals (Clarendon, Oxford, 1985).

[23] R. M. Walser, Proc. SPIE 4467, 1 (2001).

[24] H. C. van de Hulst, Light Scattering by Small Particles (Dover, New York, 1981), Sec. 6.4.

[25] I. J. Hodgkinson and Q. H. Wu, Birefringent Thin Films and Polarizing Elements (World Scientific, Singapore, 1998).

[26] A. Mondal, B. K. Mahajan, B. Choudhuri, A. K. Tiwari, S. Chakrabarty, and K. K. Chattopadhyay, J. Nanophoton. 8, 083069 (2014).

[27] W. S. Weiglhofer, A. Lakhtakia, and B. Michel, Microw. Opt. Technol. Lett. 15, 263 (1997); Corrections: 22, 221 (1999).

[28] L. Tsang and J. A. Kong, Radio Sci. 16, 303 (1981).

[29] N. P. Zhuck, Phys. Rev. B 50, 15636 (1994).

[30] E. David, Zeit. Phys. 114, 389 (1939).

[31] D. N. Jarrett and L. Ward, J. Phys. D 9, 1515 (1976).

[32] M. Born and E. Wolf, Principles of Optics, 6th ed. (Cambridge University Press, Cambridge, England, 1980), Sec. 4.5.2.

[33] T. G. Mackay and A. Lakhtakia, Modern Analytical Electromagnetic Homogenization (IOP, Bristol, 2015).

[34] T. G. Mackay, Waves Random Media 14, 485 (2004); Corrections: Waves Random Complex Media 16, 85 (2006).

[35] V. Myroshnychenko and C. Brosseau, J. Appl. Phys. 97, 044101 (2005).

[36] X. Zhao, Y. Wu, Z. Fan, and F. Li, J. Appl. Phys. 95, 8110 (2004).

[37] A. Akyurtlu and D. H. Werner, IEEE Trans. Antennas Propag. 52, 3273 (2004). 